\title{
Una mirada positivista a los desacuerdos teóricos*
}

\section{Theoretical Disagreements through Positivistic Eyes}

\author{
Jordi Ferrer Beltrán ${ }^{* *}$ y Giovanni Battista Ratti***
}

Recepción y evaluación de propuesta: 24/10/2017

Aceptación: 26/02/2018

Recepción y aceptación final: 16/08/2018

\begin{abstract}
Resumen: Los autores analizan las partes de Legalidad ${ }^{1}$ que abordan el problema de los desacuerdos jurídicos y la conexa teoría de la interpretación (y metainterpretación) desplegada por Shapiro con el fin de superar tal dificultad. La cuestión de los desacuerdos jurídicos, junto con las principales asunciones del positivismo jurídico, hace necesario, de acuerdo con los autores, prestar cierta atención a las versiones contemporáneas de tal concepción teórico-jurídica. En consecuencia, la estructura del artículo es la siguiente: en el primer párrafo, se trata el positivismo en el modo en que normalmente es explicado en la teoría del derecho angloamericana; en el segundo párrafo, los autores presentan su propia explicación del positivismo jurídico metodológico, deudora en gran medida de las obras de Bobbio y Ross; en el tercer párrafo, se resume el argumento de los desacuerdos y se analizan atentamente las dificultades que supuestamente plantea al positivismo jurídico; esto lleva al cuarto párrafo, donde la concepción de Shapiro de la interpretación jurídica y su consiguiente respuesta a semejante desafío son examinadas;
\end{abstract}

Traducción de Miguel Fernández Núñez.

** Profesor de Filosofía del Derecho y Director de la Cátedra de Cultura Jurídica, Universitat de Girona (España). E-mail: jordi.ferrerb@udg.edu

${ }^{* * *}$ Profesor de Filosofía del Derecho, Università degli Studi di Genova (Italia). E-mail: gbratti@unige.it.

1 Shapiro, S., Legalidad, Madrid-Barcelona, Marcial Pons, 2014. 
en el quinto y último párrafo, se extraen algunas conclusiones, siendo la principal de ellas que la sofisticada teoría de la interpretación de Shapiro es, por un lado, supererogatoria y, por otro, infiel al auténtico espíritu del positivismo metodológico tradicional, al mezclar aspectos descriptivos y prescriptivos de la interpretación jurídica.

Palabras clave: positivismo jurídico, metodología, interpretación, desacuerdos jurídicos.

\begin{abstract}
The authors analyze the portions of Legalidad which deal with the problem of legal disagreements and the related theory of interpretation (and metainterpretation) deployed by Shapiro in order to overcome that difficulty. The question of legal disagreements, together with the main assumptions of legal positivism, according to the authors, makes it necessary to pay some attention to the current accounts of such a jurisprudential conception. As a consequence, the structure of their article is this: in the first section, it deals with legal positivism in the way it is commonly accounted for in Anglo-American jurisprudence; in the second section, the authors present their own account of methodological legal positivism, much indebted to Bobbio's and Ross's works; in the third section, the argument from disagreements is summed up and the difficulties it allegedly poses to legal positivism are carefully analyzed; this leads to the fourth section, where Shapiro's conception of legal interpretation and consequent response to such a challenge is examined; in a fifth and final section, some conclusions are drawn, the main of which is that Shapiro's sophisticated theory of interpretation is, on the one hand, supererogatory, and, on the other hand, unfaithful to the genuine spirit of traditional methodological positivism, since it conflates descriptive and prescriptive aspects of legal interpretation.
\end{abstract}

Keywords: legal positivism, methodology, interpretation, legal disagreements. 


\section{El debate angloamericano sobre el positivismo jurídico (en pocas palabras)}

Las ideas de Shapiro en torno al positivismo jurídico están fuertemente arraigadas en el debate angloamericano entre las dos escuelas cismáticas del positivismo jurídico incluyente (en lo sucesivo, PJI) y del positivismo jurídico excluyente (PJE): en el ámbito de dicho debate, Shapiro es un conocido partidario del segundo ${ }^{2}$.

Con el fin de comprender mejor la teoría de Shapiro, es aconsejable mencionar, aunque de forma sucinta, el debate entre defensores del PJI y del PJE ${ }^{3}$.

En la teoría jurídica angloamericana, el positivismo jurídico se articula a menudo en torno a dos tesis principales, la "tesis de las fuentes sociales" (social thesis) y la "tesis de la separabilidad" (separability thesis) ${ }^{4}$. La primera afirma que aquello que cuenta como derecho en una cierta comunidad social es, principalmente, un problema de hechos sociales. La segunda declara que el derecho como es y el derecho como debe ser son dos cuestiones distintas. Las dos tesis son, con frecuencia, leídas conjuntamente. $\mathrm{O}$, mejor dicho, la tesis de la separabilidad es leída a través de las gafas de la tesis de las fuentes sociales ${ }^{5}$. El debate

2 Cfr. Shapiro, S., "On Hart's Way Out", en Coleman, J. (ed.), Hart's Postscript, Oxford, Oxford University Press, 2001.

3 Leiter, B., Naturalismo y teoría del derecho, Madrid, Marcial Pons, 2012, p. 108. Shapiro, S., Legalidad, op. cit., p. 331, explica el debate en términos de lo que llama "tesis del carácter último" (Ultimacy Thesis) -que afirma que "los hechos jurídicos, en última instancia, están determinados exclusivamente por hechos sociales" y que es defendida tanto por el PJE como por el PJI- y la "tesis del carácter excluyente" (Exclusivity Thesis) -según la cual "los hechos jurídicos están determinados exclusivamente por hechos sociales" y que es defendida solo por el PJE-.

4 La tercera afirmación iuspositivista -la llamada "tesis de la discrecionalidad" (Discretion Thesis)-, que normalmente acompaña a las otras dos tesis mencionadas en el texto, será analizada al final del presente párrafo. Es oportuno notar que el debate en la teoría del derecho de los países de civil law también se basa, al menos en parte, en las tesis examinadas, a pesar de que su interpretación difiera de forma significativa de la asumida generalmente en el área de common law. Cfr., entre muchos, Bulygin, E., El positivismo jurídico, México D. F., Fontamara, 2006.

5 Como veremos, tal lectura es engañosa, dado que estas comprenden distintos niveles de investigación acerca del derecho. 
teórico-jurídico, de hecho, ha tenido por objeto la cuestión "de si la tesis de las fuentes sociales debería ser interpretada en el sentido de formular simplemente las condiciones de existencia de una regla de reconocimiento [...] o si la tesis de las fuentes sociales formula también un vínculo sobre el contenido del test de validez jurídica que toda regla de reconocimiento puede establecer" 6 . Como es sabido, el PJI sostiene la primera lectura, mientras que el PJE defiende la segunda.

Las distintas interpretaciones de la tesis de las fuentes sociales (propugnadas, respectivamente, por el PJI y por el PJE) están, por tanto, vinculadas a la cuestión de si el derecho está separado o no de la moral. De hecho, el PJI defiende la tesis según la cual "no es necesariamente cierto que en todo sistema jurídico la validez jurídica de una norma dependa de su valor moral", mientras que el PJE defiende la tesis en virtud de la cual "es necesariamente cierto que la validez jurídica de una norma no depende de su valor moral"7. Es decir, para el PJI, el derecho puede estar "ontológicamente" conectado con (así como separado de) la moral, mientras que para el PJE el derecho no puede presentar nunca tal conexión.

Shapiro ha ofrecido uno de los intentos más conocidos de defender el PJE a partir del presupuesto de que un sistema jurídico "inclusivo" no puede cumplir la función de guiar el comportamiento humano. $\mathrm{Su}$ reciente libro Legalidad marca una nueva etapa en su sofisticada defensa del PJE. En efecto, una de las principales tesis del libro es

6 Leiter, B., op. cit., pp. 107-108.

7 Cfr. Coleman, J., "Second Thoughts and Other First Impressions", en Bix, B. (ed.), Analyzing Law. New Essays in Legal Theory, Oxford, Oxford University Press, 1998, p. 265. (La traducción de las obras citadas es del traductor de este texto, salvo que se indique otra cosa). Nótese que, en la formulación de Coleman, la tesis del PJE implica la del PJI, en el momento en que "necesario" implica "posible". No obstante, se trata de una implicación sumamente contraintuitiva. Para evitarla, la tesis del PJI debe ser reformulada del siguiente modo: "No es necesariamente cierto que en todo sistema jurídico la validez de una norma dependa de su valor moral ni que la validez jurídica de una norma no dependa de su valor moral". Esto significa que es contingente que la validez jurídica de una norma dependa de sus méritos morales. Por cuanto "contingente" y "necesario" son dos modalidades incompatibles, tenemos ahora una explicación que no comporta la consecuencia contraintuitiva de que el PJE implique el PJI. Debemos a Jorge Rodríguez esta clarificación. 
que los planes inhiben la deliberación respecto de los hechos que pretenden regular. Un "plan", según Shapiro ${ }^{8}$, es un tipo de norma que reduce los "costes deliberativos" en caso de incertidumbre. El sistema jurídico, a su vez, es una organización que produce planes, dirigidos a corregir los defectos morales relativos a las cuestiones morales que son advertidas como particularmente complejas y/o controvertidas en una determinada comunidad política. Este es, según Shapiro, el rasgo distintivo del derecho'.

Ahora bien, según la "teoría del derecho como plan", si queremos que el derecho guíe el comportamiento, no podemos admitir referencia alguna a la moral, ya que ello haría necesario deliberar nuevamente sobre los hechos que han inducido a dotarse de un plan maestro y volvería tal plan y el haberse valido de un sistema jurídico completamente inútiles ${ }^{10}$.

Se trata, como es evidente, de una reformulación del argumento ya clásico de Shapiro según el cual el PJE es conceptualmente preferible al PJI justamente por el hecho de que el segundo no sería apto para dar cuenta de la función de guía de la conducta propia del derecho, ya que la regla de reconocimiento del PJI no sería capaz de marcar la diferencia práctica en el proceso deliberativo (ni desde un punto de vista epistémico ni desde un punto de vista motivacional).

Otro punto interesante de la presentación del debate entre el PJI y el PJE proporcionada por Shapiro es su discusión de la "tesis de la

8 Shapiro, S., Legalidad, Ibidem, p. 169-171. Para una presentación más amplia de la noción de "plan", ver en este mismo número el artículo de Pino, cfr. Pino, G, “¿Cuál es el plan? Sobre la interpretación y la meta interpretación en Legalidad de Scott Shapiro", en Discusiones, XX(2), 2017.

9 Shapiro, S., ibidem, p. 265. Críticas asimilables a esta visión (denominada por Shapiro "la tesis del fin moral") en Gardner, J., Macklem, T., "Review of S. J. Shapiro's 'Legality'", Notre Dame Philosophical Reviews, 2011.

${ }^{10}$ Shapiro, S., ibidem, p. 168-169, n. 5: "cuando quien planifica reconoce que su plan es aplicable, no debería deliberar sobre los méritos del caso en cuestión. Se supone que el plan resuelve si se debe actuar de cierto modo y, por tanto, deliberar antes de la ejecución socava el propósito fundamental del plan. El planificador ejecuta racionalmente el plan porque lo adopta y es aplicable, y por ninguna otra razón". 
discrecionalidad". Las ideas de Shapiro al respecto pueden ser resumidas del siguiente modo:

1) Siendo un artefacto humano, el derecho es limitado y no puede resolver todas las situaciones jurídicamente relevantes ${ }^{11}$;

2) el PJI y el PJE proporcionan explicaciones distintas de aquello que hacen los jueces en los casos no resueltos por normas basadas en hechos sociales: mientras que el PJI sostiene que los jueces pueden recurrir a estándares morales, válidos en virtud de la regla de reconocimiento, el PJE les niega tal posibilidad;

3) en consecuencia, para el PJI "los jueces estarían identificando el derecho incluso cuando llevaran a cabo un razonamiento moral, porque estarían utilizando normas que son seleccionadas en el nivel más alto por algún hecho social" 12 .

4) Para el PJE, en cambio, tal cosa no es posible, ya que cualquier norma carente de pedigrí no puede ser una norma jurídica. Según el PJE, "el argumento sobre los límites de lo social implica que el derecho contiene muchas lagunas e inconsistencias no resueltas, y que los jueces no tienen más remedio que actuar como legisladores" 13 o aplicar normas externas al sistema en cuyo ámbito operan ${ }^{14}$.

Estamos sustancialmente de acuerdo con la reconstrucción, ofrecida por Shapiro, del comportamiento judicial según el PJI y el PJE

11 Ibidem, p. 327.

12 Ibidem, p. 333.

13 Ibidem, p. 334.

14 Ídem: "los positivistas jurídicos excluyentes están de acuerdo con la observación de Dworkin de que los jueces estadounidenses generalmente asumen que hay normas que resuelven los casos difíciles y que tienen la obligación jurídica de descubrirlas y aplicarlas. Pero arguyen que Dworkin ha malinterpretado los hechos: el comportamiento judicial en los casos difíciles no muestra que el formalismo sea verdadero y que los jueces carezcan de discreción fuerte. En los casos difíciles, en que las normas primarias con pedigrí se han agotado, los jueces estadounidenses simplemente tienen una obligación jurídica de aplicar estándares extra-jurídicos. En otras palabras, el hecho de que los jueces estadounidenses tengan la obligación de aplicar normas sin pedigrí no implica que estén obligados a aplicar derecho preexistente; en lugar de ello, sencillamente tienen una obligación de ir más allá del derecho y aplicar las normas de la moral". 
respectivamente. Sin embargo, es conveniente plantear alguna observación adicional.

En primer lugar, parece que Shapiro sostiene que los sistemas morales son siempre determinados, al afirmar que las lagunas normativas se pueden colmar fácilmente recurriendo a las normas morales. No pretendemos aquí ahondar en la cuestión, pero permítasenos sugerir al menos que resulta sumamente dudoso que los sistemas morales ofrezcan siempre una única respuesta a cualquier cuestión jurídica. De veras, no es difícil imaginar o rastrear distintos sistemas morales incompletos ${ }^{15}$.

En segundo lugar, parece también que Shapiro sostiene que los sistemas de derecho positivo, de acuerdo a cualquier tipo de positivismo, son necesariamente incompletos: lo cual es bastante sorprendente. Hay muchos pasajes en que Shapiro defiende esta tesis:

es imposible que los hechos sociales seleccionen un conjunto completo de reglas para todos los casos concebibles. Las normas con pedigrí frecuentemente se agotarán, dejando muchas lagunas e inconsistencias no resueltas. $\mathrm{Y}$, como los positivistas [excluyentes] niegan que las normas morales carentes de pedigrí sean derecho, no pueden completar el vacío jurídico ${ }^{16}$.

Por otro lado, el positivismo jurídico excluyente acepta el argumento de Dworkin de que el positivismo jurídico se compromete con el antiformalismo moderado. El derecho está determinado exclusivamente por hechos sociales y, puesto que los hechos sociales no pueden resolver toda cuestión por adelantado, el derecho contendrá muchas lagunas y contradicciones ${ }^{17}$.

El derecho está completamente determinado, entonces, cuando regula toda acción bajo toda posible descripción. El derecho

15 Es asimismo posible que, en caso de laguna, se disponga de distintos sistemas morales. En ausencia de un metacriterio ordenador, escoger una solución moral, caso por caso, de distintos sistemas morales en competición conduciría muy probablemente al surgimiento de antinomias.

16 Ibidem, p. 327.

${ }^{17}$ Ibidem, pp. 335-336. 
está indeterminado, en cambio, si no regula alguna acción bajo alguna descripción posible. Esto ocurrirá en una gran cantidad de casos, incluyendo aquellos en que la acción se ubica en la zona de penumbra de alguna regla, pero no en el núcleo de una regla más específica; en el núcleo de una regla moralmente cargada, pero no en el núcleo de una regla más específica moralmente neutra; o en el núcleo de dos reglas inconsistentes no existiendo una regla que resuelva tales conflictos. Puesto que las conductas están comprendidas en una de esas categorías de manera inevitable, se infiere que el derecho nunca está completamente determinado ${ }^{18}$.

La tesis del carácter "necesariamente incompleto" del derecho elaborada por Shapiro presenta algunas dificultades. A nuestro juicio, la cuestión de si son completos los sistemas jurídicos es una cuestión empírica, no conceptual. No es conceptualmente imposible (pese a ser empíricamente arduo) que una autoridad normativa legisle de modo exhaustivo respecto de un cierto ámbito de relaciones sociales. Una vez que se ha identificado el universo del discurso relevante, la autoridad normativa tiene la posibilidad de regular de forma exhaustiva las acciones que caen en el ámbito de tal universo. Shapiro, al examinar el PJE, parece rechazar esta posibilidad cuando afirma que "es imposible que los hechos sociales seleccionen un conjunto completo de reglas para todos los casos concebibles" porque los "hechos sociales no pueden resolver toda cuestión por adelantado". Si "concebibles" significa "concebibles en el ámbito de un cierto universo del discurso", no se ve por qué ello deba considerarse imposible. Las autoridades normativas pueden resolver, en un nivel abstracto en que se hace referencia a clases de acciones o eventos, todas las cuestiones por adelantado: lo que no pueden hacer, a causa de la vaguedad (intensional y extensional), es determinar completamente las decisiones judiciales en el nivel particular de la aplicación (o sea, en el nivel de los casos individuales) ${ }^{19}$.

18 Ibidem, p. 344.

${ }^{19}$ Alchourrón, C. E., Bulygin, E., Sistemas normativos. Introducción a la metodología de las ciencias jurídicas (1971), Buenos Aires/Bogotá, Astrea, 2012, p. 47 y ss; Alchourrón, C. E., "On Law and Logic" (1996), en Ferrer Beltrán, J., Ratti, G. B. (eds.), The Logic of Legal Requirements. Essays on Defeasibility, Oxford, Oxford University Press, 2012. 
La principal razón conceptual por la que Shapiro considera que el derecho es necesariamente incompleto puede residir en la tesis por la cual el derecho está completamente determinado solo cuando regula toda acción bajo toda posible descripción. Por lo que se puede entender, su argumento es el siguiente: puesto que las acciones pueden ser descritas en infinitas maneras, las autoridades normativas, que pueden gestionar solo un número limitado de descripciones de estados de cosas, no pueden imaginar y disciplinar todas ellas. El argumento, reconstruido de este modo, no convence. En primer lugar, las autoridades normativas podrían formular una norma general de clausura (que normalmente consiste en una norma permisiva ${ }^{20}$ para todos los posibles universos de discurso (aunque no conozcan todas sus posibles descripciones) ${ }^{21}$. Ello volvería el derecho completo bajo toda posible descripción, pero lo volvería también incoherente, ya que los casos más específicos (o sea, los casos identificados por medio de un conjunto más rico de propiedades relevantes) resultarían conectados con soluciones incompatibles con las asignadas a casos menos específicos (o sea, casos identificados mediante un conjunto más restringido de propiedades ${ }^{22}$. Con todo, los

${ }^{20}$ Otras normas de clausura-como "Todo lo que no está cualificado jurídicamente es obligatorio" o "Todo lo que no está cualificado jurídicamente está prohibido"-crean dificultades lógicas de sobra conocidas. Cfr., de nuevo, Alchourrón, C. E., Bulygin, E., op. cit., p. 190 y ss.

${ }^{21}$ De hecho, lo que hace posible la legislación mediante normas generales y abstractas es la ley lógica del refuerzo del antecedente (también llamado "principio de aumentación"), según la cual un enunciado condicional implica un condicional cuyo antecedente originario está enriquecido por la adición de una proposición cualquiera. En lenguaje simbólico: $(\mathrm{p} \supset \mathrm{q}) \supset(\mathrm{p} \& \mathrm{r} \supset \mathrm{q})$. Así es que las autoridades normativas, por definición, no pueden concebir todas las combinaciones futuras de las propiedades, pero pueden disciplinarlas precisamente mediante la regla del refuerzo del antecedente.

22 Alchourrón, C. E., Bulygin, E., op. cit., pp. 149-150 y 243 y ss. Para aclarar el punto, consideremos un conocido ejemplo. Una norma dispone que, en el caso de que se esté en presencia de un testamento válido y de la muerte del testador por parte del heredero, esté prohibido al heredero recibir el caudal hereditario (en lenguaje simbólico: “w \& k つ O i "). Sin embargo, el caso menos fino caracterizado por la sola presencia de un testamento válido no es, en hipótesis, resuelto por una determinada norma del sistema (recuérdese que las soluciones dispuestas por normas más específicas no son aplicables, por razones lógicas, a normas menos específicas). Por consiguiente, podemos aplicar a dicho caso la norma de clausura permisiva: se sigue de ello que ahora 
sistemas jurídicos normalmente proporcionan soluciones para ciertos universos del discurso (lo que quiere decir que las autoridades normativas no están interesadas en disciplinar todos los posibles universos del discurso): esto significa que no son concebidos para resolver cada caso bajo cualquier descripción posible (la definición de "determinación completa" introducida por Shapiro, aun siendo interesante desde un punto de vista lógico, es irrelevante para los sistemas reales). Tales sistemas son concebidos, más bien, para resolver determinadas cuestiones en la descripción jurídicamente relevante. Si esto es correcto, no se comprende por qué un sistema jurídico no pueda nunca ser completo.

Otro argumento a favor de una indeterminación radical del sistema propuesto por Shapiro consiste en señalar que los casos pueden caer en una de las siguientes categorías: (1) en la zona de penumbra de una norma pero no en el núcleo de una norma más específica, (2) en el núcleo de una norma moralmente cargada pero no en el núcleo de una norma moralmente neutra más específica, o (3) en el núcleo de dos normas antinómicas. Dado que esto es inevitable, Shapiro concluye que "el derecho nunca está completamente determinado"23.

Conviene notar que las categorías usadas por Shapiro son conceptualmente controvertidas. En primer lugar, las primeras dos categorías tienen que ver con casos individuales, y no genéricos: es decir, se refieren a la subsunción de casos particulares en normas generales. Pero tal subsunción puede resultar dudosa aunque el derecho sea completo respecto de las clases de acciones o eventos que aspira a regular.

La tercera categoría, obviamente, debe quedar circunscrita en un doble sentido: en primer lugar, se debe distinguir entre antinomias que pueden ser resueltas y antinomias que no pueden ser resueltas por metacriterios; en segundo lugar, conviene distinguir entre antinomias

resulta conectado con el permiso de heredar ("w $\supset \sim \mathrm{O} \sim \mathrm{i}$ "). Es posible concluir que el caso caracterizado por la presencia de un testamento válido y la muerte del testador a manos del heredero está conectado con dos soluciones incompatibles (prohibido/ permitido).

${ }^{23}$ La cita completa es la siguiente: "Puesto que las conductas están comprendidas en una de esas categorías de manera inevitable, se infiere que el derecho nunca está completamente determinado" (Shapiro, S., ibidem, p. 344). 
por contrariedad (p.e., $\mathrm{Op} \& \mathrm{O} \sim \mathrm{p}$ ) y antinomias por contradictoriedad (p.e., Op \& Op), siendo solo las primeras efectivamente irresolubles desde un punto de vista práctico ${ }^{24}$. Se debe notar asimismo que el juez, cuando se enfrenta con antinomias aplica siempre una norma válida al caso (mediante una ordenación jerárquica previa) ${ }^{25}$, de tal modo que no es del todo correcto hablar del "agotamiento" del derecho, o de su indeterminación, entendida como insuficiencia de recursos.

Por estas razones, no se puede dar por sentado en ningún modo que el positivismo jurídico presuponga, o implique, que el derecho es necesariamente indeterminado desde un punto de vista sistemático. Evidentemente, esto no excluye que el derecho pueda ser indeterminado desde el punto de vista interpretativo: dicho de otro modo, la tesis según la cual no es necesario que el derecho sea indeterminado desde un punto de vista sistemático no implica en modo alguno la tesis por la cual no es necesario que el derecho sea indeterminado desde el punto de vista interpretativo.

\section{El positivismo jurídico y la reafirmación de la tesis de la separación}

La lectura del positivismo jurídico que comúnmente brinda el debate angloamericano, si bien es muy influyente, nos parece bastante engañosa y sustancialmente contraria a los orígenes modernos del positivismo jurídico, tal y como se encuentran en los trabajos de Jeremy Bentham y John Austin, y como han sido defendidas, más recientemente, con instrumentos filosóficos parcialmente renovados, por Norberto Bobbio y Alf Ross.

${ }^{24}$ En el caso de antinomias por contradictoriedad, el destinatario hace bien, desde un punto de vista práctico, en actuar la obligación de hacer $p$ y en no usar el permiso de abstenerse de hacer $p$ (o, en el caso de prohibiciones, en actuar la obligación de no hacer $p$ y en no usar el permiso de hacer $p$ ).

${ }^{25}$ Gavazzi, G., Studi di teoria del diritto, Turín, Giappichelli, 1993, p. 145. 
En particular, sostendremos que la llamada "tesis de la separación" 26 es incomprendida sistemáticamente en el ámbito de dicho debate ${ }^{27}$.

El positivismo jurídico, a nuestro juicio, puede ser caracterizado de modo (cuando menos) dúplice ${ }^{28}$ :

1) Desde un punto de vista metodológico, el positivismo jurídico constituye la tesis epistemológica por la cual el derecho puede ser conocido en términos estrictamente empíricos, basándose en la observación de ciertos hechos sociales ${ }^{29}$. En otras palabras, es la afirmación en virtud de la cual el derecho puede ser descrito de forma valorativamente neutra: este es el núcleo de la lectura epistémica de la tesis de la separación.

(2) Desde un punto de vista teórico, el positivismo jurídico puede ser concebido como la reconstrucción racional de tales (contingentes) hechos sociales.

Estos dos aspectos, aunque conectados en la práctica, no deben ser confundidos en la teoría.

${ }^{26}$ Preferimos la expresión "tesis de la separación" a la expresión "tesis de la separabilidad", porque la última evoca una ontología de los mundos posibles que está en total contraste con la ontología fuertemente empirista implícita en la lectura epistémica de esta tesis, que se expresa mejor en referencia a la neta separación (y no solo separabilidad) de los dominios de hechos y valores.

${ }^{27}$ Ross, A., "El concepto de validez y el conflicto entre el positivismo jurídico y el derecho natural" [1961], en Ross, A., El concepto de validez y otros ensayos, México, Fontamara, 1993, p. 13, señala que es "en alto grado engaños[o]" concebir la tesis de la separación como una tesis sustancial u ontológica sobre la separación entre derecho y moral. Para una opinión contraria, que, sin embargo, no tiene en cuenta el tratamiento rossiano, cfr. Green, L., "Positivism and the Inseparability of Law and Morals", New York Law Review, 83, 2008, p. 1038: "La tesis de la separabilidad no es una afirmación metodológica. Solo rige en el dominio del nivel-objeto -esto es, en leyes y sistemas jurídicos".

${ }^{28}$ Bobbio, N., Giusnaturalismo e positivismo giuridico, Milán, Comunità, 1965, p. 101-126; Ross, A., "Validity and the Conflict between Positivism and Natural Law", op. cit., p. 148-149.

${ }^{29}$ Según Ross (op. cit., p. 150), el "grito de batalla" de Austin, en virtud del cual la existencia del derecho es una cosa y sus méritos o defectos otra, debe ser entendido en este sentido. 
El primer modo, metodológico, de concebir el positivismo (la versión epistémica de la tesis de la separación, opuesta a la versión metafísica de tal tesis o tesis de la separabilidad) se ha perdido casi del todo en el debate angloamericano. Esto puede sonar sorprendente, en el momento en que gran parte de la formación teórico-jurídica en los países de common law se basa precisamente en el análisis de la tesis de la separación. Sin embargo, dicha tesis es leída como una afirmación sustantiva, y no epistémica. En efecto, toda la discusión entre partidarios del PJI y defensores del PJE, que hemos referido poco antes, gira en torno a la cuestión de si el derecho puede o no incorporar la moral. Pero esta parece una mera cuestión de "etiquetas", que atañe a lo que se pretende denominar "derecho" 30 . La propuesta del PJE, en particular, parece una mera estipulación del término "derecho", cuya consecuencia principal consiste en negar el título de "derecho" a aquellos sistemas de normas sociales que pretenden incorporar, o hacer referencia a, la moral ${ }^{31}$. Obviamente, es posible que las autoridades normativas busquen incorporar, o remitir a, la moral (rectius, a un determinado sistema de normas morales), con lo que no queda claro en que consiste la ventaja de negar el título de "derecho" a tales sistemas de normas ${ }^{32}$.

${ }^{30}$ Shapiro, S., Legalidad, Ibidem, p. 336: "que el debate entre el positivismo excluyente e incluyente es esencialmente una disputa de ese tipo [terminológico]. Después de todo, el punto de discusión es si resulta adecuado llamar 'norma jurídica' a una norma sin pedigrí que los jueces tienen la obligación jurídica de aplicar. En otras palabras, tanto el positivista excluyente como el incluyente coinciden en que los jueces tienen la obligación de aplicar normas morales una vez se agotan las normas con pedigrí. Simplemente, discrepan acerca de cómo describir lo que están haciendo: para el positivista jurídico incluyente, los jueces están aplicando normas jurídicas; para el positivista jurídico excluyente, están creando normas jurídicas".

31 Así entendido, el PJE se asemeja mucho, en la estructura lógica, al iusnaturalismo definitorio, sobre el cual véase Celano, B., "Giusnaturalismo, positivismo giuridico e pluralismo ético", Materiali per una storia della cultura giuridica, XXXV/1, 2005. Cfr. asimismo Ratti, G. B., Diritto, indeterminatezza, indecidibilità, Madrid, Marcial Pons, 2012, p. 45. En lo sucesivo, daremos otra lectura del PJE.

${ }^{32}$ Frederick Schauer ha observado, en una comunicación privada, que podríamos apresurarnos demasiado al sostener que el positivismo jurídico excluyente se limita a estipular una definición de "derecho". La definición propuesta por el PJE capta una realidad empírica en la cual las disposiciones jurídicas, los libros de derecho, las facul- 
El PJI, por su parte, ha debilitado tanto la tesis de la separación como para desproveerla de todo atractivo teórico. El positivismo jurídico, de acuerdo con los defensores del PJI, estaría a salvo al sostener que el derecho y la moral son separables en al menos un mundo posible. Sin embargo, esta tesis admite, a contrario, que el derecho y la moral (objetiva) no están casi nunca separados en el mundo real: por tanto, es difícil comprender por qué razón deberíamos continuar usando el término "positivismo" para una tesis en tan fuerte contraste con los datos empíricos.

Contrariamente a estas ideas, el positivismo jurídico, entendido como metodología, establece vínculos solo al método con el que adquirir conocimiento jurídico genuino, pero no a los hechos que constituyen fuentes del derecho. La tesis de la separación -el núcleo de tal metodología- no es otra cosa que la siguiente afirmación: el derecho puede ser conocido de forma valorativamente neutra. Lo que quiere decir que, desde un punto de vista epistemológico, el derecho puede ser conocido

tades de derecho, etc., ocupan un universo empírico separado del universo moral (pese a que los límites sean difusos). En efecto, aunque aludamos a la conexión empírica entre derecho y moral, hay igualmente maneras importantes en que dichos ámbitos normativos son empíricamente diferentes. Concordamos con la observación de Schauer, pero somos de la opinión de que tal separación empírica concierna eminentemente a la "institucionalización" de las fuentes del derecho, no a su interpretación ni a las potenciales "referencias" que tales fuentes puedan realizar, con mayor o menor éxito, a la moral. A nuestro juicio, el PJE, al menos en la influyente versión Raziana, no tiene los instrumentos necesarios para ofrecer una explicación de los fenómenos atinentes a la interpretación y al drafting legislativo que hemos mencionado hace un momento. Sería necesaria una versión distinta del PJE, por así decir "depurada", que defendiese el carácter meramente factual, valorativamente neutro, de la identificación de las fuentes $y$ una visión moderadamente escéptica de la interpretación jurídica (que no niega necesariamente -como sugiere la teoría formalista de la interpretación normalmente asociada con el PJE- relaciones interpretativas entre el significado de las fuentes jurídicas y consideraciones axiológicas o morales). Para una teoría de la interpretación excluyente y formalista, de estricto credo raziano, véase Marmor, A., Interpretation and Legal Theory, Oxford, Hart, 2005, p. 95, quien sostiene que: "el positivismo jurídico no puede aceptar la concepción de que la ley está siempre sujeta a interpretación. Sencillamente no puede ser que toda conclusión sobre lo que es el derecho sea resultado de una interpretación u otra". 
como un mero conjunto de hechos, sin que sea necesaria, con el fin de conocerlo, valoración alguna.

Lo que, en cambio, impone vínculos a las fuentes del derecho es, en nuestra opinión, la concepción de la metaética que subyace a las distintas concepciones del derecho. En nuestra opinión, la única concepción plausible de la metaética es el no-cognitivismo. En el momento en que los hechos morales objetivos no existen, las posibles referencias a tales entidades morales efectuadas por las autoridades normativas deben considerarse como intentos fallidos de incorporar normas morales objetivas en un cierto sistema jurídico o imponer a los jueces la aplicación de normas que pertenecen a otro sistema normativo (en este caso, inexistente).

No es este el lugar para preparar una defensa articulada del no-cognitivismo ético. Con todo, la razón principal que nos lleva a defenderlo puede ser formulada de forma sencilla. Sobre la base de los métodos científicos difundidos en nuestra comunidad, podemos sostener que no existe prueba alguna a favor de la existencia de hechos morales objetivos. Cuando no existe ninguna prueba a favor de una aserción, se tiene licencia para pensar que tal aserción es falsa, cuando menos en el nivel de la inducción $n^{33}$. Esto es exactamente lo que ocurre con las presuntas verdades morales objetivas: no hay prueba alguna de su existencia; pero, puesto que, conforme a nuestros estándares científicos, consideramos falso lo que no puede contar con ninguna prueba tenemos fuertes razones para considerar que el objetivismo ético es una tesis falsa. Se sigue que el derecho no puede sino consistir solo en hechos empíricos, por la muy prosaica razón de que solo tales hechos existen. Si es así, el PJI, así como el dworkinismo, son, en el mejor de los casos, simples exposiciones de lo que los operadores jurídicos creen que hacen y no de lo que realmente hacen. En particular, el PJI es solo un modo de afirmar que las autoridades normativas en ocasiones pretenden incorporar normas morales objetivas, independientemente

${ }^{33} \mathrm{E} 1$ argumento ad ignorantiam constituye una muy conocida falacia deductiva. Con todo, es también sabido que la investigación científica y la obtención de pruebas tienen una estructura eminentemente inductiva. 
de la cuestión de si tal concepción resulta o no exitosa (a nuestro juicio, tales intentos están necesariamente destinados al fracaso $)^{34}$.

Desde el punto de vista teórico, en cambio, el positivismo jurídico puede ser considerado una exposición (o, mejor, una reconstrucción) de los hechos sociales de los que está constituido el derecho. No se trata de una investigación de la "esencia" o de la "naturaleza" del derecho (investigación que caracteriza las doctrinas del derecho natural) ${ }^{35}$, sino de una investigación concerniente a las formas y las estructuras contingentes que los sistemas jurídicos pueden asumir.

En este contexto teórico, y tras haber sido depurados de todo elemento esencialista, el PJI y el PJE pueden considerarse (o, mejor, deberían ser reformulados) como empresas teóricas, que buscan explicar tales formas y estructuras contingentes. A pesar de que diverjan en innumerables aspectos de la reconstrucción teórica del derecho, hay una asunción principal que es particularmente importante para el análisis que realizaremos en lo sucesivo. La "tesis nuclear" de ambos es que el derecho se basa en el acuerdo o en la convención: o sea, los hechos sociales, a los que hace referencia la tesis del carácter último (Ultimacy Thesis), consisten, fundamentalmente, en prácticas constituidas por comportamientos convergentes basados en el consenso.

Manteniendo separadas estas dos perspectivas sobre el positivismo jurídico (la metodológica y la teórica), es posible examinar de forma fructífera los desacuerdos jurídicos, ya que el desafío de Dworkin tal vez pueda menoscabar el positivismo teórico (especialmente aquel tipo de positivismo que explica el derecho, o su existencia, en términos de acuerdo $)^{36}$, pero no invalida de ningún modo el positivismo metodo-

${ }^{34}$ Cfr. Priel, D., "Farewell to the Exclusive-Inclusive Debate", Oxford Journal of Legal Studies, 25, 2005.

${ }^{35}$ Este modo de concebir la teoría jurídica -como señala Guastini, R., Distinguiendo. Estudios de teoría y metateoría del derecho, Barcelona, Gedisa, 1999, p. 22- no es fácilmente separable del iusnaturalismo, puesto que presupone la idea (típicamente iusnaturalista) de que todos los sistemas jurídicos comparten algunas propiedades necesarias, independientemente de su colocación espacio-temporal.

${ }^{36}$ Esto es más que dudoso. Sobre el asunto, cfr. Dolcetti, A., Ratti, G. B., "Los desacuerdos jurídicos y la 'doble naturaleza' del derecho”, en Luque Sánchez, P., Ratti, G. B. 
lógico (i.e., la tesis de que el derecho es susceptible de ser conocido científicamente, en términos empíricos) ${ }^{37}$.

\section{El argumento de los desacuerdos como presunta refutación del positivismo jurídico}

La segunda parte del libro de Shapiro está articulada, en buena medida, en torno al argumento de los desacuerdos propuesto por Dworkin como una presunta refutación del positivismo jurídico. En pocas palabras, el conocido argumento dworkiniano es el siguiente.

Dworkin utiliza dos dicotomías para atacar el positivismo jurídico en el tema de los desacuerdos en el derecho ${ }^{38}$.

La primera dicotomía está constituida por la pareja "fundamentos del derecho" (grounds of law) y "proposiciones de derecho" (propositions of law). Las segundas son proposiciones que vierten sobre la existencia de una cierta norma en un determinado sistema jurídico. Las primeras constituyen lo que hace verdaderas las proposiciones de derecho. Qué son los fundamentos del derecho o cómo se conciban depende, obviamente, de las opciones teóricas que suscriba cada autor. En verdad, una de las discusiones principales de la teoría jurídica vierte sobre la cuestión de si los hechos morales pueden, o incluso deban, aparecer entre las condiciones de verdad de las proposiciones de derecho.

La segunda dicotomía atañe a la naturaleza de los posibles desacuerdos sobre el derecho. Un primer tipo de desacuerdo (que Dworkin llama "empírico") versa sobre la cuestión de si las circunstancias necesarias para la existencia jurídica de ciertos fundamentos del derecho hayan tenido lugar efectivamente (por ejemplo: si una determinada ley ha sido aprobada con la mayoría prevista por el reglamento parlamentario). Un segundo tipo de desacuerdo (que Dworkin llama "teórico")

(eds.), Acordes y desacuerdos. Cómo y por qué los juristas discrepan, Madrid, Marcial Pons, 2013, pp. 179-202.

37 Bobbio, N., op. cit., pp. 124-126.

${ }^{38}$ Dworkin, R., Law's Empire, Londres, Fontana, 1986, pp. 3-6. 
consiste en una divergencia referente a cuáles son los fundamentos del derecho. Se está frente a un desacuerdo teórico, por ejemplo, toda vez que distintos jueces tengan opiniones divergentes sobre la cuestión de si los estándares normativos sociales (la constitución, las leyes, las decisiones judiciales, etc.) agotan los fundamentos del derecho relevantes, o no.

Como observa de forma convincente Shapiro ${ }^{39}$, una de las principales tesis del "segundo" Dworkin consiste en sostener que:

en la concepción de los meros hechos, los desacuerdos teóricos son imposibles. La razón es sencilla: [...] un hecho $f$ es un fundamento del derecho solo si existe acuerdo entre los funcionarios en que es un fundamento del derecho. Los desacuerdos entre los funcionarios sobre si $\mathrm{f}$ es un fundamento del derecho son, por consiguiente, incoherentes: sin consenso acerca de si f es un fundamento del derecho, $f$ no es un fundamento del derecho. Podríamos decir que, según la concepción de los meros hechos, los desacuerdos teóricos se auto-refutan. [...] Los desacuerdos coherentes sobre el derecho solo pueden ser relativos a afirmaciones en conflicto sobre la existencia o inexistencia de meros hechos históricos. En otras palabras, deben ser desacuerdos empíricos.

Como hemos notado en otras ocasiones ${ }^{40}$, con el fin de comprender y desmitificar el desafío de Dworkin, es preciso distinguir distintos tipos de desacuerdos en ámbito jurídico. Esto se debe al hecho de que la expresión "fundamentos del derecho" es usada por Dworkin de modo ambiguo y muchos de los argumentos articulados por Dworkin en tema de desacuerdos sobre las bases del derecho están aquejados de falacias de equivocación.

La expresión "fundamentos del derecho" designa, en un primer sentido, las posibles fuentes del derecho (i. e., constituciones, leyes,

${ }^{39}$ Shapiro, S., ibidem, p. 350.

${ }^{40}$ Ratti, G. B., "Los desacuerdos jurídicos en la jurisprudence anglosajona", Analisi e diritto, 2008, pp. 301-331. Para una clasificación más articulada, cfr. Dolcetti, A., Ratti, G. B., op. cit. 
decisiones judiciales, principios de derecho natural, etc.), mientras que en un segundo sentido denota el contenido de significado de dichas fuentes. Como es obvio, pueden producirse desacuerdos sobre cuáles son las fuentes del derecho o sobre cuál es su significado.

En pos de la claridad, parece oportuno redefinir como "desacuerdos de reconocimiento" aquellos desacuerdos que proceden de las distintas teorías que los jueces (y, más en general, los juristas) usan para identificar las fuentes del derecho. Por "fuente del derecho" entendemos aquí cualquier formulación normativa (o sea, cualquier enunciado reductible, sin pérdida de significado, a un enunciado deóntico) que puede ser utilizado por los órganos judiciales para motivar su decisión ${ }^{41}$.

Se puede convenir en llamar, en cambio, "desacuerdos interpretativos" aquellas divergencias que tienen que ver con la validez, la ordenación o el uso de distintos cánones interpretativos, que deben ser usados al atribuir un significado a las distintas fuentes del derecho.

Obviamente, es posible hipotetizar vínculos entre desacuerdos de reconocimiento y desacuerdos interpretativos. Sin embargo, ello es completamente contingente: un desacuerdo en el nivel de la teoría de las fuentes del derecho, efectivamente, no implica de ningún modo un desacuerdo relativo al contenido de significado de tales fuentes y viceversa.

${ }^{41}$ Con ello reformulamos parcialmente la noción de fuente jurídica ofrecida por Ross, A., Sobre el derecho y la justicia (1958), Buenos Aires, Eudeba, 1963, p. 75: "Por 'fuentes del derecho', pues ha de entenderse el conjunto de factores o elementos que ejercen influencia en la formulación, por parte del juez, de las reglas en que éste basa su decisión". Esto permite considerar como posibles fuentes jurídicas no solo los documentos normativos, sino también el "derecho vivo" (i.e., el conjunto de normas que surgen "espontáneamente" en el ámbito de una cierta comunidad jurídica) o las normas derivadas (i.e., aquellas normas que constituyen el producto de inferencias lógicas, o pseudológicas, a partir de normas formuladas). Empero, que tales normas carentes de formulación asuman, eventualmente, el valor de fuente jurídica se debe al hecho de que un órgano jurídicamente competente las haya formulado en la que se considera su forma canónica. Esta (amplia) definición de "fuente" permite cualificar como fuentes jurídicas "objetos" tan dispares como los documentos normativos, los principios morales, las normas consuetudinarias y los precedentes judiciales: lo que parece ser presupuesto por el debate anglosajón sobre los fundamentos del derecho. 
A nuestro parecer, la distinción entre "desacuerdos de reconocimiento" y "desacuerdos interpretativos" disuelve toda la cuestión planteada por Dworkin, ya que el positivismo jurídico solamente debe demostrar que, en general, existe un acuerdo (rectius, una convergencia identificativa) respecto de cuáles son las fuentes del derecho, y no necesariamente acerca de cuál es su interpretación ${ }^{42}$.

Esto se puede demostrar fácilmente si pensamos en dos situaciones distintas: probablemente, podamos afirmar que existe una regla de reconocimiento si hay un acuerdo (o al menos una convergencia de hecho) acerca de cuál sea la principal fuente de un sistema jurídico (por ejemplo, la constitución), aun cuando la interpretación de tal fuente resulte controvertida. En cambio, no se puede sostener de forma sensata que exista un sistema jurídico si los jueces discrepan, de forma extensa, acerca de cuál es la fuente principal en que se funda el sistema.

En el momento en que los desacuerdos teóricos de los que habla Dworkin tienen que ver con desacuerdos sobre el significado ${ }^{43}$, y no con las fuentes, el positivismo se encuentra a salvo al sostener que un cierto tipo de consenso cimenta la existencia del derecho ${ }^{44}$.

${ }^{42}$ Hart, H. L. A., Postscriptum al concepto de derecho, México D. F., Universidad Nacional Autónoma de México, 2000, p. 47: "Ciertamente, la regla de reconocimiento es tratada en mi libro como reposando en una forma convencional de consenso judicial. Que efectivamente ésta descanse así, parece muy claro, al menos en el derecho inglés y en el derecho estadounidense porque, ciertamente, la razón de un juez inglés para tratar a la legislación del Parlamento (o la razón de un juez estadounidense para tratar la Constitución) como una fuente de derecho que tiene supremacía sobre todas las otras fuentes incluye el hecho de que sus colegas de la judicatura concurren en esto como sus predecesores lo hicieron" (cursiva nuestra). De esta cita, resulta claro que Hart sostiene que la regla de reconocimiento, que es el producto del consenso judicial, versa sobre las fuentes del derecho, antes que sobre su significado. Cfr., en cualquier caso, Ratti, G. B., "Regla de reconocimiento, cánones interpretativos y realismo jurídico", en Luque Sánchez, P., Ratti, G. B. (eds.), op. cit., pp. 315-328.

${ }^{43}$ Esta observación la insinúan los propios ejemplos que Dworkin escoge para atacar el positivismo jurídico y la reconstrucción de estos casos que proporciona en Law's Empire. Sobre el punto, cfr. Leiter, B., "Cómo explicar los desacuerdos entre juristas", en Luque Sánchez, P., Ratti, G. B. (eds.), op. cit., pp. 75-109.

${ }^{44}$ Debemos observar, de pasada, que, a nuestro juicio, el derecho no está necesariamente basado sobre un cierto tipo de acuerdo o consenso. En consecuencia, desde una perspectiva teórica, adherimos completamente a la afirmación de Schauer, F., "The best laid 
Sin embargo, frente a nuestra opinión, la conclusión que se puede derivar, manifiestamente, del tratamiento de los desacuerdos ofrecido por Shapiro en Legalidad es que lo que los positivistas quieren decir (o deberían querer decir, si quisieran reconstruir el positivismo de manera adecuada) con "acuerdo" es tanto "acuerdo sobre las fuentes" como "acuerdo sobre su interpretación". Para que exista un sistema jurídico ambos son necesarios.

Shapiro, por ejemplo, afirma que ${ }^{45}$ :

Los debates sobre el método interpretativo correcto plantean una dificultad todavía mayor para el positivismo jurídico. Como sostuvo Ronald Dworkin, el mero hecho de que tales disputas tengan lugar indica que el derecho no puede fundamentarse en el tipo de hechos que el positivista cree que conforman la base de los sistemas jurídicos. Los positivistas han afirmado que los criterios de validez jurídica están determinados por convención y consenso. Pero los debates sobre la metodología interpretativa demuestran que tal convención o consenso no existe. En otras palabras, los desacuerdos sobre el método interpretativo son imposibles según la posición del positivista jurídico. Sin embargo, no solo parecen posibles, sino que tienen lugar de modo generalizado. Como puede apreciarse, estas objeciones ejemplifican otra forma de desafío desde el razonamiento jurídico. Conforme a esta versión, los positivistas jurídicos no pueden dar cuenta de un cierto tipo de desacuerdo que mantienen con frecuencia quienes razonan jurídicamente, a saber, desacuerdos concernientes al método correcto para interpretar el derecho. La única explicación plausible de cómo esos desacuerdos son posibles, prosigue esta versión de la objeción, es que son disputas políticas que pueden ser resueltas solo

plans", Yale Law Yournal, 120, 2011, p. 621, según la cual: "Con demasiada frecuencia, el libro de Shapiro queda atrapado en un milieu teórico que desatiende la penetración generalizada de la coerción y exagera la importancia de la posibilidad, decididamente contrafáctica, de un derecho libre de sanciones". Sea como fuere, lo que sostenemos en el texto es que quienes creen que el derecho está basado en el consenso tienen una vía de escape muy simple del presunto puzle formulado por Dworkin.

45 Shapiro, S., ibidem, pp. 346-347. 
mediante el razonamiento moral. En contra del positivismo jurídico, el derecho no se basa y no puede basarse solo en hechos sociales, sino que se fundamenta en última instancia en consideraciones de moral política.

Y, de nuevo ${ }^{46}$ :

Los debates sobre si los textos jurídicos deberían interpretarse de manera estricta o amplia, de acuerdo con sus significados públicos originarios, costumbres sociales que evolucionan, tradiciones fuertemente arraigadas, la intención de los constituyentes, según los resultados esperados, o los principios morales, con deferencia a la interpretación judicial previa, a los órganos de la administración, a la doctrina, o a las leyes extranjeras, o en conjunción con las historia legislativa o disposiciones similares, o aisladamente, todos estos, son fenómenos absolutamente normales en muchos sistemas jurídicos modernos. Dworkin ha señalado que el positivismo jurídico, al menos como se lo concibe actualmente, no puede dar sentido a esta verdad obvia y por tanto es incapaz de dar cuenta de un aspecto central de la práctica jurídica.

Anticipando el resultado principal de nuestra discusión, podemos afirmar que no estamos de acuerdo con Shapiro sobre este tema. Los motivos principales son los siguientes.

El positivismo metodológico se limita a indagar el derecho como un dato empírico: desde esta perspectiva, la afirmación de que el derecho es lo que de hecho es no excluye que entre tales hechos puedan encontrarse también las (eventualmente divergentes) valoraciones de legisladores, jueces y juristas ${ }^{47}$.

Esto, a su vez, significa que puede darse el caso (aunque no sea necesario) de que los desacuerdos se propaguen y que cualquier cuestión requiera de una valoración interpretativa para ser decidida. En este caso, el positivista metodológico debe limitarse a tomar nota de la
${ }^{46}$ Ibidem, pp. 355-356.
${ }^{47}$ Bobbio, N., op. cit., p. 124. 
circunstancia de que hay algunos hechos que conforman las fuentes del derecho y otros hechos que constituyen las (divergentes) valoraciones de los juristas sobre el significado de dichas fuentes.

Desde un punto de vista teórico, una vez que se haya asumido la relevancia de la noción de acuerdo, no se puede sino dar cuenta de los principales casos de acuerdo y de desacuerdo en un sistema jurídico e intentar articular una explicación completa de aquellos en cuanto características del sistema jurídico. Y aquí, una vez más, la que nos parece la mejor explicación es que los sistemas jurídicos generalmente se caracterizan por un acuerdo relativamente extenso sobre las fuentes y parciales (aunque penetrantes, al menos en los más altos niveles jurisdiccionales) desacuerdos sobre su interpretación.

Evidentemente, para dar cuenta de tales divergencias interpretativas, es necesaria una teoría de la interpretación jurídica. En la medida en que el positivismo jurídico angloamericano no ha desarrollado una teoría de la interpretación acabada ${ }^{48}$, no debe sorprender que no pueda explicar los desacuerdos acerca de la interpretación jurídica.

No obstante, Shapiro ha colmado dicha laguna y ha desarrollado, en los últimos capítulos de su libro, una sofisticada y articulada teoría de la interpretación que merece un análisis cuidadoso y nos induce a seguir elaborando nuestra concepción de la interpretación. A ello dedicaremos el próximo apartado ${ }^{49}$.

\section{Shapiro sobre la interpretación jurídica}

En Legalidad, Shapiro introduce una interesante dicotomía entre interpretación y metainterpretación, con el fin de afrontar las críticas de Dworkin sobre los desacuerdos. La interpretación determina una metodología específica para interpretar los textos jurídicos, mientras que

${ }^{48}$ Leiter, B., Naturalismo y teoría del derecho, op. cit., pp. 116-118; Guastini, R., L'interpretazione dei documenti normativi, Milán, Giuffrè, 2004, pp. 57-61.

49 En adelante, trataremos solo de aquellos aspectos de la teoría de la interpretación de Shapiro que consideramos fundamentales para el análisis de las controversias jurídicas. Para un análisis más exhaustivo, véase de nuevo Pino, G., op. cit. 
la metainterpretación establece una metodología para determinar qué metodología interpretativa es correcta ${ }^{50}$.

Si nuestra lectura de Shapiro es correcta, "interpretación" denota una doctrina prescriptiva que establece cómo se debe atribuir significado a los textos jurídicos: por ejemplo, "las disposiciones jurídicas deben ser interpretadas literalmente" o "las disposiciones jurídicas deben ser interpretadas según la intención del legislador", etc.

La expresión "metainterpretación", por su parte, designa una doctrina prescriptiva que determina qué teoría interpretativa es correcta: por ejemplo, "la metodología interpretativa correcta es aquella que hace eficaces los propósitos de quienes han formulado el plan", o "la metodología interpretativa correcta es aquella que hace que el sistema aparezca en su mejor luz moral".

Con estos (nuevos) conceptos disponibles, Shapiro reformula el argumento de Dworkin de los desacuerdos teóricos del siguiente modo ${ }^{51}$ :

La concepción de los meros hechos resulta ser una teoría meta-interpretativa. Sostiene que la metodología interpretativa se determina por la metodología aceptada por todos los funcionarios de justicia de un sistema particular. El problema con la concepción de los meros hechos, como Dworkin señala, es que elimina la posibilidad de disputas meta-interpretativas. $\mathrm{Si}$ los funcionarios discrepan sobre la metodología interpretativa, entonces, de acuerdo con la concepción de los meros hechos, no existe una metodología interpretativa adecuada. Sin embargo, como los desacuerdos meta-interpretativos no son solo posibles sino frecuentes, la concepción de los meros hechos no puede ser una teoría meta-interpretativa adecuada.

Esta reformulación del desafío de Dworkin, en un primer momento no es tan fácil de captar. A nuestro parecer, comúnmente se asume que la concepción factual (que no es otra cosa que la denominación dworkiniana del positivismo jurídico excluyente) es (o cuando menos

\footnotetext{
${ }^{50}$ Shapiro, S., op. cit., p. 370.

51 Ibidem, p. 371.
} 
pretende ser) una teoría descriptiva: o sea, no es una doctrina sobre cómo debería ser interpretado el derecho, es (o aspira a ser) más bien una exposición teórica de cómo es el derecho. El hecho de que los juristas no estén de acuerdo sobre las doctrinas metainterpretativas, y tampoco sobre las doctrinas interpretativas, no contrasta con el PJE, como nosotros lo entendemos, por cuanto este busca solo explicar cuáles son los criterios de validez jurídica y los vínculos existentes en relación con ellos.

Si los jueces en general consideran que "el derecho como integridad" es la doctrina metainterpretativa correcta, el PJE -como lo concebimos nosotros- sugiere que se tome nota de tal circunstancia y que sea explicada como un hecho social y nada más ${ }^{52}$.

Si los jueces no comparten una única doctrina metainterpretativa o interpretativa, los iuspositivistas excluyentes aún podrían explicar la situación a examen en los términos de un (parcial) acuerdo.

En primer lugar, existe un acuerdo acerca de quiénes son los jueces. En segundo lugar, existe un acuerdo sobre cuáles son las fuentes jurídicas; en caso contrario, ninguna divergencia metainterpretativa o interpretativa tendría sentido. De cualquier modo, nos parece que el positivismo jurídico excluyente, interpretado correctamente, no tiene nada que decir sobre cuál sea la interpretación correcta, si esta es entendida en sentido prescriptivo. Está solo interesado en decir algo, desde un punto de vista descriptivo y detached, sobre lo que es considerado "correcto" por quienes participan en la práctica jurídica.

Dicho esto, es cierto que el PJE es reconstruido habitualmente como una teoría que tácitamente implica o presupone una cierta doctrina normativa de la interpretación. Curiosamente, no obstante, no es la doctrina que Shapiro evoca en Legalidad. El PJE, al menos en la formulación de Raz y del propio Shapiro en trabajos precedentes, está caracterizado por la idea de que la deliberación práctica de los desti-

${ }^{52}$ Shapiro (op. cit., p. 460) parece ser consciente de ello, en el punto en que afirma: "Que algún conjunto de objetivos y valores represente los propósitos de un determinado sistema jurídico es un hecho acerca de ciertos grupos sociales que puede determinarse mediante un razonamiento empírico, en lugar de moral". 
natarios normativos debe ser anticipada por las normas jurídicas. Esto, a su vez, significa que los destinatarios normativos no pueden usar, si se quiere que el sistema cumpla su función de motivar a los asociados y hacer conocibles sus posiciones jurídicas, aquellos cánones interpretativos que hacen referencia a las presuntas razones subyacentes a las reglas (pues ello implicaría una nueva deliberación sobre las cuestiones morales que el derecho pretende resolver). Se sigue de ello que la reconstrucción del PJE ofrecida por Shapiro conduce a un dilema: o la metodología metainterpretativa correcta es fijada por el acuerdo (pero en este caso, en el supuesto de que se acepten cánones interpretativos de índole teleológica, no está garantizado que las reglas puedan funcionar como razones excluyentes) o la metodología metainterpretativa correcta es fijada por la esencial función propia del derecho de cerrar el paso a la deliberación moral de sus destinatarios (y, en este caso, serían admitidos solo cánones simultáneamente literales y "originalistas"). Podemos por tanto distinguir dos metodologías propias del PJE: una metodología metainterpretativa basada en el consenso y una metodología interpretativa basada en las razones excluyentes ${ }^{53}$.

En Legalidad, sin embargo, Shapiro rechaza una metodología basada en el consenso en virtud del hecho de que las controversias entre juristas abundan ${ }^{54}$.

la concepción de los meros hechos, o cualquier otra concepción que privilegie las convenciones interpretativas como la única fuente para la metodología adecuada, debe ser rechazada. Puesto que los desacuerdos teóricos abundan en el derecho, la metodología interpretativa debe ser fijada de modo distinto que por el acuerdo social específico sobre qué metodologías son adecuadas.

Lo que Shapiro parece afirmar es que la metodología basada en el consenso (nótese, una doctrina aparentemente prescriptiva de la inter-

${ }^{53}$ Es preciso notar que puede darse el caso de que las dos metodologías sean coextensivas: esto ocurre cuando la metodología aceptada habitualmente es la literal-originalista. En verdad, ocurre muy raramente. 
pretación $)^{55}$ no siempre es útil para identificar el conjunto correcto de técnicas interpretativas, puesto que son frecuentes los desacuerdos metainterpretativos. Cada vez que haya desacuerdos en torno a la interpretación, deben ser empleadas otras doctrinas metainterpretativas.

Tras rechazar una metodología basada en el consenso, Shapiro parece optar por una metodología basada en las razones excluyentes.

Descrita en detalle, la respuesta de Shapiro al desafío de Dworkin se basa en una particular teoría de la "confianza", que puede ser resumida sucintamente con una simple máxima: cuanta mayor confianza se deposite en un sujeto más libertad interpretativa le será reconocida, y viceversa ${ }^{56}$. No nos resulta muy claro si la tesis de Shapiro pretende ser una descripción de lo que ocurre realmente, una regla técnica sobre aquello que debería ocurrir si se asume cierta proposición anankástica como verdadera, o solo una recomendación al legislador acerca de la estructura de los ordenamientos jurídicos.

55 Ibidem, p. 370: "La denominó teoría de la meta-interpretación puesto que no establece una metodología específica para interpretar los textos jurídicos, sino una metodología para determinar qué metodología específica es adecuada. En otras palabras, proporciona a los participantes de los sistemas concretos los recursos necesarios para determinar si han de suscribir el literalismo, el constitucionalismo vivo, el originalismo, el pragmatismo, el derecho como integridad, etc.". Esta formulación parece compatible con una interpretación explicativa: la metainterpretación no es la elección de los cánones interpretativos, sino un presupuesto de ella (aquello que la hace posible). Con todo, en otros pasajes, Shapiro defiende claramente una perspectiva prescriptiva sobre la metainterpretación: "la Teoría de los planes sostiene, con Dworkin, que en tales casos la metodología interpretativa adecuada para un sistema jurídico particular es principalmente una función de qué metodología promovería mejor los objetivos que el sistema pretende alcanzar" (ibidem, p. 459).

56 Ibidem, p. 403: "la Teoría de los planes conlleva que las actitudes de confianza y desconfianza presupuestas por el derecho son centrales a efectos de la elección de una metodología interpretativa. A grandes rasgos, la Teoría de los planes exige que cuanto más confiable se considere que es una persona, más discreción interpretativa se le confiere; a la inversa, cuanto menos se confía en alguien en otras esferas de la vida jurídica, menos discreción se le concede. Sostengo que las actitudes de confianza son centrales para la metainterpretación del derecho porque son centrales para la meta-interpretación de los planes - y las normas jurídicas son planes, o normas similares a planes". 
En todo caso, una vez rechazada una metodología basada en el consenso como la metodología correcta, es preciso determinar los recursos que permiten identificar ese otro método (sin consentir una nueva deliberación sobre las cuestiones que el derecho pretende resolver). En lo tocante a este punto, Shapiro afirma ${ }^{57}$ :

La metodología interpretativa adecuada se establece determinando qué metodología armoniza mejor los objetivos establecidos por los planificadores del sistema [...].

La postura de Shapiro con respecto a la metodología metainterpretativa parece ser claramente prescriptiva, en el momento en que impone a los intérpretes la obligación de reconstruir los objetivos que tenían los planners al construir el sistema jurídico de referencia.

Con todo, curiosamente, Shapiro considera que esta doctrina prescriptiva tiene consecuencias explicativas o descriptivas ${ }^{58}$ :

La virtud de este tipo de propuesta es que, en la medida en que la metodología interpretativa no tiene que estar determinada por una convención específica sobre la metodología adecuada, es capaz de dar cuenta de la posibilidad de que haya desacuerdos teóricos. Los participantes en una práctica pueden discrepar sobre la metodología interpretativa adecuada porque no están de acuerdo sobre si su práctica puede ser mejor descrita como un sistema autoritativo u oportunista y, por tanto, a los juicios de quién deberían deferir.

Dicho esto, Shapiro concluye ${ }^{59}$ :

Nótese además que esta teoría es positivista. Puesto que adopta la ideología que inspira un régimen como su piedra de toque, esta reconstrucción puede terminar recomendando una metodología interpretativa basada en un conjunto de creencias y valores moralmente cuestionables. El sistema jurídico en cuestión, por ejemplo, puede

57 Ibidem, p. 460.

58 Ídem. 
existir para promover la desigualdad racial o la intolerancia religiosa; puede plasmar concepciones ridículas acerca de la naturaleza humana y los límites del conocimiento. Sin embargo, el intérprete positivista adopta esta ideología como dada, y trata de determinar qué metodología interpretativa armoniza mejor con ella.

Si no erramos en la comprensión del significado de este pasaje, parece que la doctrina de Shapiro no es en absoluto una teoría puramente descriptiva de la interpretación (y por tanto no puede dar cuenta, de forma valorativamente neutra, de un fenómeno determinado), es, más bien, una ideología de la interpretación que impone a los intérpretes actuar su (presunta) concepción moral de un sistema jurídico. Esta parece ser una versión de positivismo ético o ideológico ${ }^{60}$. Si es así, pertenece a un tipo de discurso distinto de aquel del positivismo metodológico y teórico que hemos delineado previamente.

Desde el punto de vista de la política del derecho, rechazamos con fuerza una obligación general de obedecer al derecho o de realizar la moral de un sistema jurídico (independientemente de sus méritos).

Sea como fuere, no nos interesa de ningún modo formular una doctrina normativa de la interpretación jurídica; antes bien, pretendemos dedicarnos a una teoría del derecho genuinamente descriptiva y valorativamente neutra: desde este enfoque, no podemos dispensarnos de observar que la reconstrucción de la interpretación que ofrece Shapiro no es puramente descriptiva. Por esta razón, no puede formar parte de una teoría puramente descriptiva del derecho y, cosa todavía más importante para nuestros fines actuales, no puede explicar en lo más mínimo las controversias jurídicas (puede solo recomendar cómo resolverlas, una vez que hayan sido identificadas). 


\section{Conclusiones}

Las conclusiones principales que se pueden extraer de lo que hemos sostenido hasta ahora son las siguientes:

1) El debate sobre el positivismo jurídico típico de la teoría jurídica angloamericana confunde distintos aspectos de tal concepción que deberían mantenerse separados. En particular, la tesis de la separación es leída, por los participantes en dicho debate, sea como una verdad analítica sobre el derecho sea como una afirmación ontológica extraordinariamente débil, mientras que es posible entenderla, de forma más caritativa, como una afirmación epistémica fuerte.

2) La objeción dworkiniana de los desacuerdos puede ser rechazada con facilidad tanto desde un punto de vista metodológico como desde un punto de vista teórico. Desde un enfoque metodológico, los desacuerdos sobre la interpretación de las fuentes no son problemáticos, ya que un defensor del positivismo metodológico puede limitarse a tomar nota de ellos como simples hechos. Desde un punto de vista teórico, el positivismo jurídico, en la versión que intenta explicar el derecho en términos de consenso, puede ser defendido en la medida en que explica el derecho en términos de un acuerdo relativamente amplio sobre las fuentes, pero no necesariamente sobre su significado.

3) La teoría de la interpretación elaborada por Shapiro con el fin de afrontar la crítica de Dworkin nos parece, por un lado, supererogatoria, y, por otro, infiel al espíritu auténtico del positivismo tradicional. Es supererogatoria porque semejante cantidad de sofisticación filosófica y de esfuerzo intelectual no es, a nuestro juicio, necesario para responder a la objeción de Dworkin. Y es infiel al positivismo en el momento en que confunde indebidamente aspectos descriptivos y justificativos de la interpretación jurídica. 
Una mirada positivista a los desacuerdos teóricos

\section{Bibliografía}

Alchourrón, C. E., "On Law and Logic" (1996), en Ferrer Beltrán, J., Ratti, G. B. (eds.), The Logic of Legal Requirements. Essays on Defeasibility, Oxford, Oxford University Press, 2012.

Alchourrón, C. E., Bulygin, E., Sistemas normativos. Introducción a la metodología de las ciencias jurídicas (1971), Buenos Aires,Bogotá, Astrea, 2012.

Bobbio, N., Giusnaturalismo e positivismo giuridico, Milán, Comunità, 1965.

Bulygin, E., El positivismo jurídico, México D. F., Fontamara, 2006.

Celano, B., "Giusnaturalismo, positivismo giuridico e pluralismo etico", Materiali per una storia della cultura giuridica, XXXV, 1, 2005.

Coleman, J., "Second Thoughts and Other First Impressions", en Bix, B. (ed.), Analyzing Law. New Essays in Legal Theory, Oxford, Oxford University Press, 1998.

Dolcetti, A., Ratti, G. B., "Los desacuerdos jurídicos y la "doble naturaleza" del derecho", en Luque Sánchez, P., Ratti, G. B. (eds.), Acordes y desacuerdos. Cómo y por qué los juristas discrepan, Madrid, Marcial Pons, 2013, pp. 179-202.

Dworkin, R., Law's Empire, Londres, Fontana, 1986.

Gardner, J., Macklem, T., "Review of S. J. Shapiro's 'Legality”, Notre Dame Philosophical Reviews, 2011.

Gavazzi, G., Studi di teoria del diritto, Turín, Giappichelli, 1993.

Green, L., "Positivism and the Inseparability of Law and Morals", New York Law Review, 83, 2008.

Guastini, R., Distinguiendo. Estudios de teoría y metateoría del derecho, Barcelona, Gedisa, 1999.

Guastini, R., L'interpretazione dei documenti normativi, Milán, Giuffrè, 2004.

Hart, H. L. A., Post scríptum al concepto de derecho, México D. F., Universidad Nacional Autónoma de México, 2000.

Leiter, B., Naturalismo y teoría del derecho, Madrid, Marcial Pons, 2012. Leiter, B., "Cómo explicar los desacuerdos entre juristas", en Luque Sánchez, P., Ratti, G. B. (eds.), Acordes y desacuerdos. Cómo y por qué los juristas discrepan, Madrid, Marcial Pons, 2013, pp. 75-109. 
Jordi Ferrer Beltrán / Giovanni Battista Ratti

Marmor, A., Interpretation and Legal Theory, Oxford, Hart, 2005.

Pino, G, "¿Cuál es el plan? Sobre la interpretación y la meta interpretación en Legalidad de Scott Shapiro", Revista Discusiones, XX(2), 2017.

Priel, D., "Farewell to the Exclusive-Inclusive Debate", Oxford Journal of Legal Studies, 25, 2005.

Ratti, G. B., "Los desacuerdos jurídicos en la jurisprudence anglosajona", Analisi e diritto, 2008, pp. 301-331.

Ratti, G. B., Diritto, indeterminatezza, indecidibilità, Madrid, Marcial Pons, 2012.

Ratti, G. B., "Regla de reconocimiento, cánones interpretativos y realismo jurídico", en Luque Sánchez, P., Ratti, G. B. (eds.), Acordes $y$ desacuerdos. Cómo y por qué los juristas discrepan, Madrid, Marcial Pons, 2013, pp. 315-328.

Ross, A., Sobre el derecho y la justicia (1958), Buenos Aires, Eudeba, 1963.

Ross, A., "El concepto de validez y el conflicto entre el positivismo jurídico y el derecho natural" (1961), en Ross, A., El concepto de validez y otros ensayos, México, Fontamara, 1993

Schauer, F., "The best laid plans", Yale Law Yournal, 120, 2011.

Shapiro, S., "On Hart's Way Out", en Coleman, J. (ed.), Hart's Postscript, Oxford, Oxford University Press, 2001.

Shapiro, S., Legalidad, Madrid-Barcelona, Marcial Pons, 2014, traducción de Papayannis, D. M. y Ramírez Ludeña, L. 